\title{
EDITORIAL.
}

\section{The Ethics of Criticism.}

Of all the functions of editorial work that of the reviewer demands most care and conscientious attention, while it is so generally slighted as to have ceased to command respect or confidence.

A few general principles would seem to be self-evident, which nevertheless may be worth formulating afresh.

1. The writer of a scientific paper may reasonably be supposed to be actuated primarily by a desire for the advancement of his science and his effort should receive recognition at least to that extent.

2. The writer should be assumed to be a gentleman and should be treated as such until he has notoriously forfeited his claim to be so considered.

3. The mistakes or oversights for which the writer is responsible must be considered unintentional until avowed by him.

4. Criticism should emphasize the commendable features of a writer's work if any exist.

5. Personalities are utterly and absolutely out of the question in scientific reviewing.

6. A review may avoid all critical intent by confining itself to an abstract but such abstract must then be adequate and not misleading unless it be explicitly stated that only one aspect of the paper is included. The art of making a clear and competent abstract is, like that of translation, very difficult and success is rare and meritorious.

7. When a review is at the same time a critique the criticism must be explicit or where in general terms must contain illustrations of the faults pointed out. 
A general statement such as "The paper displays a painful lack of discrimination" is only permissible when accompanied by instances of such failure to discriminate. A charge that "the author has failed to avail himself of the literature of his subject" should point out some omissions of the sort described. In a word, a critique must not appear to exist for the sake of finding fault but for the sake of of correcting or refuting error and must be absolutely impersonal. Even such a statement as " the evidence for the author's position seems to be insufficient," harmless as it seems, were best either omitted or followed by an explanation of the ambiguity or insufficiency. If an author be charged with failure to make his point it is but just that he should be shown where and how he has erred.

8. It ought not to be necessary to insist that the reviewer should be held responsible for a faithful effort to understand his author. The author may be obscure but before he can be taxed with this fault the reviewer must make a reasonable effort to reach his point of view. Many long and profitless public discussions would have been prevented by a private correspondence and such a course is to be recommended where at all possible. The use of the pages of a scientific periodical for purposes which could better be served by personal enquiry is to be deprecated. It is not claimed that the writer has lived up to the principles here laid down but in so far as he has failed he expresses contrition and only adds that, in order to attain the ends above sought and to add dignity to the reviewer's position, it is believed that all extended critiques should be signed by the writer.

C. L. Herrick. 